OPEN ACCESS

Edited by:

David B. Go,

University of Notre Dame,

United States

Reviewed by:

Patrick Jacob Shamberger,

Texas A\&M University,

United States

Liang Chen,

Xi'an Jiaotong University, China

*Correspondence:

William F. Paxton

william.f.paxton@gmail.com

Specialty section:

This article was submitted to

Thermal and Mass Transport,

a section of the journal

Frontiers in Mechanical Engineering

Received: 31 July 2017 Accepted: 07 November 2017

Published: 05 December 2017

Citation:

Paxton WF, Ravipati S, Brooks MM, Howell M and Davidson JL (2017)

Thermionic Emission from

Diamond Films in Molecular

Hydrogen Environments.

Front. Mech. Eng. 3:18.

doi: 10.3389/fmech.2017.00018

\section{Thermionic Emission from Diamond Films in Molecular Hydrogen Environments}

\author{
William F. Paxton ${ }^{1,2 *}$, Srikanth Ravipati ${ }^{1,2}$, Morgan M. Brooks ${ }^{1,3}$, Mick Howell ${ }^{4}$ \\ and Jim L. Davidson ${ }^{4}$
}

${ }^{1}$ IOP Technologies, LLC., Louisville, KY, United States, ${ }^{2}$ Conn Center for Renewable Energy Research, University of Louisville, Louisville, KY, United States, ${ }^{3}$ Ochsner Clinical School, New Orleans, LA, United States, ${ }^{4}$ Department of Electrical Engineering, Vanderbilt University, Nashville, TN, United States

Diamond-based low-work function thermionic electron emitters are in high demand for applications ranging from electron guns and space thrusters to electrical energy converters. A key requirement of such diamond-based electron sources is hydrogen termination of the surfaces which can significantly reduce the emission barrier. However, at high temperatures $\left(\leq 600^{\circ} \mathrm{C}\right)$, terminated hydrogen begins to desorb causing degradation in thermionic emission performance. The purpose of this study is to examine low-pressure hydrogen operating environments as a means to overcome this high-temperature performance limitation by enabling increased thermionic emission currents with improved stability at temperatures $\leq 600^{\circ} \mathrm{C}$. A series of isothermal and isobaric experiments were performed in both nitrogen and hydrogen gas environments to determine the performance enhancement. Diamond electron emitters in both the as-grown and hydrogenated states were characterized at temperatures of 600,625 , and $650^{\circ} \mathrm{C}$. An increase in thermionic emission current over vacuum operation was observed following the introduction of hydrogen. Upon evacuation of hydrogen to vacuum, the emission current decreased back to baseline levels. Further experiments in gas environments at a constant pressure $\left(\sim 5.5 \times 10^{-6}\right.$ Torr $)$ were conducted at temperatures ranging from 700 to $900^{\circ} \mathrm{C}$. It was observed that the hydrogen environment promoted increased emission current while also enabling the diamond electron emitters to stably emit at increased temperatures compared with vacuum operation. Analogous experiments using nitrogen environments did not show any measurable performance enhancements, thus verifying that hydrogen is responsible for the observed effect. These results suggest diamond-based electron emitters can have improved thermionic emission performance at temperatures $\leq 600^{\circ} \mathrm{C}$ when operating in hydrogen gas environments.

Keywords: thermionic emission, diamond, doped diamond, hydrogen, surface modification

\section{INTRODUCTION}

Previous research has demonstrated diamond to be an exceptional low-temperature electron emitter for numerous applications, including thermionic energy conversion (TEC). Interactions between hydrogen and diamond are credited with improving the electron emission properties. In addition to increasing the bulk conductivity of diamond films, hydrogen has been shown to 
interact with the diamond surface to form polarized $\mathrm{C}-\mathrm{H}$ bonds, reducing the electron affinity and in turn, reducing the work function (Maier et al., 2001). This effect was illustrated in previous work by Paxton et al. (2012a), which observed that exposure of diamond cathodes to a low-energy hydrogen plasma prior to testing resulted in drastically enhanced thermionic emission current relative to as-grown diamond films by four orders of magnitude.

Thermionic emission can be described by the Richardson equation seen in Eq. 1 (Richardson, 1903)

$$
J=A T^{2} e^{-\Phi / k_{b} T}
$$

where $J$ is the emission current density $\left(\mathrm{A} \mathrm{cm} \mathrm{cm}^{-2}\right), A$ the Richardson constant $\left(\mathrm{A} \mathrm{cm}^{-2} \mathrm{~K}^{2}\right), T$ the cathode temperature $(\mathrm{K}), k_{b}$ the Boltzmann constant $\left(8.617 \times 10^{-5} \mathrm{eV} \mathrm{K}^{-1}\right)$, and $\phi$ the work function of the diamond $(\mathrm{eV})$. From Eq. 1, it can be seen that thermionic emission current should follow an exponentially increasing trend with increasing temperature. However, previous studies have reported that the emission current begins to deviate from Eq. 1 at temperatures between 650 and $800^{\circ} \mathrm{C}$ in that it exhibits a decreasing trend with increasing temperature (Suzuki et al., 2009; Kataoka et al., 2010; Paxton et al., 2012a). This trend has been attributed to the desorption of hydrogen and results in a "temperature ceiling" (defined here as the temperature at which the maximum emission current is achieved) that prevents higher emission currents from being obtained. More detailed studies directly pertaining to understanding this phenomenon found that the hydrogen desorption process follows first-order kinetics; resulting in a predictable degradation in emission current during operation (Paxton et al., 2012b, 2014). In order to capitalize on diamond's favorable properties for thermionic emission applications, methods that allow such cathodes to overcome the aforementioned performance limiting factors associated with the desorption of hydrogen need to be identified.

Out of the several applications of diamond thermionic cathodes, their use in TEC is of particular interest to the authors of this study. Past implementations of such devices utilized tungsten cathodes with cesium gas fed into the cathode-anode gap (Wilson, 1959; Hatsopoulos, 1963; Hernqvist, 1963; Rasor, 1963, 1991; Witting and Gyftopoulos, 1965). With a work function greater than $4 \mathrm{eV}$, bare tungsten cathodes would require exceptionally high temperatures, greater than $2,000^{\circ} \mathrm{C}$, to emit sufficient current for a TEC device. Past TEC implementations reduced this high-temperature requirement by "cesiating" the surface of the tungsten cathodes (Wilson, 1959; Rasor, 1963, 1991). Because cesium is electropositive with respect to tungsten, it creates a polarized surface layer on the cathode, resulting in a surface with a lower work function than bare tungsten (Jenkins, 1969). The desorption of this cesium from the tungsten cathodes heated to operating temperatures was mitigated in previous implementations by incorporating cesium into the cathodeanode gap (Wilson, 1959). The motivation behind the present study was to develop a similar technique that could allow for the performance-enhancing effects of hydrogen in diamond to be constantly replenished, in situ.

There has been some previously reported work pertaining to the operation of diamond films in certain gaseous environments in an effort to improve the emission current performance. One such study by Koeck et al. (2011) examined the thermionic emission from both phosphorus doped and nitrogen-incorporated (similar to those used in the present study) diamond films in a methane ambient up to 700 mTorr at temperatures of $500^{\circ} \mathrm{C}$. While both types of diamond exhibited an increasing trend in emission current with increasing methane pressure at constant temperatures, the phosphorus doped diamond appeared to have the strongest enhancement. At temperatures of $500^{\circ} \mathrm{C}$ and methane pressure of 500 mTorr, the phosphorus doped diamond demonstrated an emission current enhancement of $\sim 3 \times$ while the nitrogen-incorporated diamond only demonstrated an enhancement of $\sim 1.6 \times$ at similar temperatures and a 700 mTorr methane pressure. The results presented in this previous study are promising but difficulties may arise during implementation, specifically in terms of stability. Drabner et al. (1990) performed a detailed study to examine the dissociation of methane as a function of pressure. It was found that at higher pressures, $>200$ mTorr, complex hydrocarbons such as of $\mathrm{C}_{2} \mathrm{H}_{\mathrm{x}}^{+}$and $\mathrm{C}_{3} \mathrm{H}_{\mathrm{x}}^{+}$are the primary product of the methane dissociation. Over extended operation periods, these molecules will likely reform on the diamond surfaces as non-diamond carbonaceous content, inevitably decreasing the performance.

It can be seen that there exist several similarities between hydrogen's interaction with diamond and cesium's interaction with tungsten. As cesium environments improved the performance and stability of tungsten cathodes, the present study sought to explore whether operation of diamond cathodes in hydrogen environments would result in a similar effect. A previously reported study by Nemanič et al. (2014) also attempted to improve the performance of diamond films through the use of hydrogen in situ. In that previous study, diamond samples that demonstrated reduced emission performance were exposed to hydrogen that was fed into the testing chamber over a heated filament. The filament was meant to introduce atomic and/ or excited molecular hydrogen, enabling it to readily interact with the diamond surface, thereby enhancing the performance. The approach used in the present study is distinct from that of Nemanič et al. in that no external source such as a heated filament was used to excite/ionize/dissociate the hydrogen, only the electrons thermioncally emitted from the diamond films. The authors believe that successful implementation of the approach presented below would prove more practical in applications such as TEC as no external energy is required to achieve the desired performance enhancement.

Results obtained in hydrogen environments were compared with identical experiments in nitrogen environments to eliminate any other variables that could influence results. Nitrogen was chosen as it has been shown to have a similar total electron scattering cross-section to hydrogen, around $10 \times 10^{-16} \mathrm{~cm}^{2}$, at the anticipated electron energies of $\sim 1 \mathrm{eV}$ (Tawara et al., 1990, Itikawa, 2006). However, unlike hydrogen, operation of diamond cathodes in nitrogen environments should have no positive effect on the thermionic emission current as its atomic state is not readily able to bond with diamond surface nor diffuse into interstitial sites due to its large atomic size. 


\section{MATERIALS AND METHODS}

Polycrystalline nitrogen-incorporated diamond films were deposited on molybdenum substrates using a microwave plasma-enhanced chemical vapor deposition (MPCVD) reactor in the manner previously described (Paxton et al., 2012a,b, 2014). Samples were deposited at $1.5 \mathrm{~kW}$ microwave power in a 50 Torr hydrogen, methane, and nitrogen environment. SEM micrographs of samples deposited under these conditions can be found elsewhere (Paxton et al., 2012a). The samples were examined in the testing configuration previously reported (Paxton et al., 2012b). A turbomolecular pump was employed that was capable of evacuating the testing chamber to a base pressure of $\sim 1 \times 10^{-7}$ Torr. The pressure was monitored by an ion gauge tube positioned on the pump rather than on the chamber to prevent interference of the gauge's heated filament with the testing of the samples.

In order to examine the operation of diamond cathodes in hydrogen and nitrogen environments, tanks of ultra-high-purity hydrogen and nitrogen were connected to the chamber via a leak valve. A residual gas analyzer (RGA) was attached to the testing apparatus for direct measurement of the gasses present in the chamber. Operation of the RGA consisted of three steps: first, the chamber was pumped for a period of $\sim 24 \mathrm{~h}$ to a base pressure of $1 \times 10^{-7}$ Torr. Second, a baseline was established by powering on the RGA with the leak valve closed and observing species present in the chamber. Finally, the hydrogen or nitrogen gas was fed into the chamber. Verification that only the desired gas was entering the chamber was made when only an $\mathrm{H}_{2}$ or $\mathrm{N}_{2}$ peak was seen during the leak-in process while all other gases remained unchanged. During testing, the RGA was powered off to prevent interference.

Two different sets of experiments were conducted to fully characterize how the hydrogen environments influenced the thermionic emission performance from the diamond cathodes. The first set consisted of isothermal testing to allow direct comparison of the emission current in a vacuum environment with that of a low-pressure hydrogen and nitrogen environments. The isobaric testing in the second set of experiments was chosen to assess how operation in a hydrogen environment could improve the stability and temperature ceiling of the diamond cathodes. Both techniques were first performed on a sample in its as-grown state. The sample was then removed from the testing chamber, hydrogenated, and tested a second time in the same manner. In all experiments, current was monitored by an RBD 9103 picoammeter with an accuracy of $\pm 0.4 \%$.

Isothermal experiments consisted of monitoring the thermionic emission current at three temperatures for an extended period of time: 600,625 , and $650^{\circ} \mathrm{C}$. Temperature control was performed by a proportional-integral-derivative control loop that maintained the temperature within $2^{\circ} \mathrm{C}$ of the target set point during testing. This temperature control has been described in detail elsewhere (Paxton et al., 2012b, 2014). An Omega IR2 2-color ratio infrared pyrometer was used to observe the temperature with an accuracy of $2^{\circ} \mathrm{C}$ across its full-scale range. At each temperature, the emission current from the sample was initially measured in vacuum $\left(\sim 1 \times 10^{-7}\right.$ Torr $)$ to establish baseline behavior. Hydrogen or nitrogen was then introduced into the chamber via the leak valve until the pressure at the pump read $\sim 5.5 \times 10^{-6}$ Torr. As mentioned, pressure was measured at the pump as the ion gauge tube operates on the principal of electron emission. Thus, attaching such a gauge directly to the chamber could affect testing results should any gauge electrons be collected by the anode. This pressure was maintained for a period of $\sim 1 \mathrm{~min}$, after which, the valve was closed, and the pressure rapidly decreased back to $1 \times 10^{-7}$ levels. This sequence was performed twice at each temperature.

The testing sequence described above was first performed on a sample (Sample A) in its as-grown state at a temperature of $600^{\circ} \mathrm{C}$ followed by two more tests at 625 and $650^{\circ} \mathrm{C}$, in order of increasing temperature. These three tests represent one testing cycle. Following completion of the third test at $650^{\circ} \mathrm{C}$ (and also the first testing cycle), the sample was allowed to cool to room temperature in vacuum and the whole process was executed again two more times, providing data from nine total tests (three testing cycles at each of the three temperatures) for the isothermal experiments. Upon completion of the ninth and final isothermal test, the isobaric experiment was initiated.

Testing for the isobaric experiment entailed observing the emission current versus temperature trend of the diamond cathode in a constant $\sim 5.5 \times 10^{-6}$ Torr $\left(\mathrm{N}_{2}\right.$ equivalent) hydrogen environment. Pressure was maintained by using the leak valve in the same manner as previously discussed for the isothermal experiments. The isobaric tests began immediately following the final $650^{\circ} \mathrm{C}$ isothermal test without allowing the sample to cool. The temperature control loop was used to increase the temperature in roughly $5^{\circ}$ increments up to $\sim 900^{\circ} \mathrm{C}$. Each temperature was maintained for $30 \mathrm{~s}$ and the emission current was constantly monitored using the same technique as previously reported (Paxton et al., 2012a). These isobaric tests were intended to determine if the presence of hydrogen would increase the emission current of diamond thermionic emitters and/or raise their operational temperature ceiling.

Following this isobaric test, the sample was cooled, re-hydrogenated in the MPCVD chamber as previously described (Paxton et al., 2012a) and both isothermal and isobaric experiments were repeated. Results obtained from the isothermal molecular hydrogen experiments were verified by conducting the same series of experiments on a second sample (Sample B) grown separately from the first but in the same conditions. Despite the natural discrepancies that inevitably occur between diamond samples grown in different batches that will influence the emission current, any beneficial effect associated with the molecular hydrogen environment should be translatable to other similar diamond samples. A third distinct sample was utilized for the nitrogen environment tests.

\section{RESULTS}

\section{Isothermal Emission Testing Results}

Emission current and pressure versus time for the as-grown sample is shown in Figure 1. Only the first testing cycle (one test at each testing temperature) is presented for publication as the other two testing cycles demonstrated similar behavior (additional data is included in the Supplementary Material section). Upon inspection, it can immediately be seen that operation in the presence 


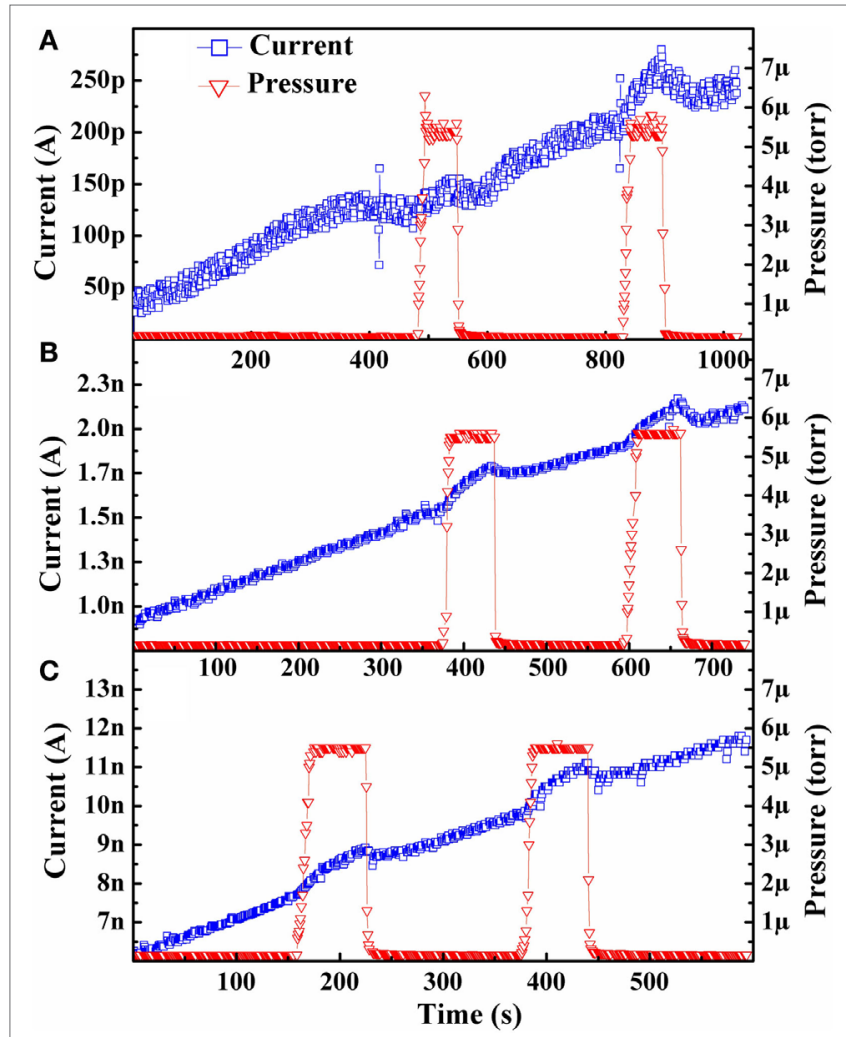

FIGURE 1 | Isothermal emission current behavior of an as-grown diamond cathode in both vacuum and low-pressure hydrogen environment at: (A) $600^{\circ} \mathrm{C}$, (B) $625^{\circ} \mathrm{C}$, and (C) $650^{\circ} \mathrm{C}$. Only the first testing cycle for the as-grown sample is presented for publication purposes as a similar trend was seen for each test in the following two testing cycles. The current and pressure data are represented by blue squares and orange triangles, respectively. of hydrogen had an enhancing effect on the emission current. As demonstrated in Figure 1, when hydrogen was fed into the chamber the emission current increased from the baseline and then subsequently decreased back to the baseline when the leak valve was closed and vacuum was reestablished.

Figure 1 also illustrates an increasing trend of the baseline emission current with respect to time for each test. This trend is somewhat contradictory to the emission behavior previously reported in Paxton et al. (2012b, 2014) where it was observed that the emission current followed a decreasing trend with time. This increase is explained by the fact that those previously reported hydrogen activation energy experiments were performed using hydrogenated (or deuterated) samples whereas the data collected in Figure 1 was performed with an as-grown sample. The hydrogenated samples had virtually all surface carbon atoms bonded with a hydrogen atom. As the sample is heated, the hydrogen desorbs from the surface into the vacuum resulting in decreasing emission. In short, when operating in a vacuum and in the absence of any other influencing factors, a fully hydrogenated diamond sample should initially be at, or close to, its maximum possible thermionic emission performance level. The reason for the lower emission from an as-grown sample, compared with a

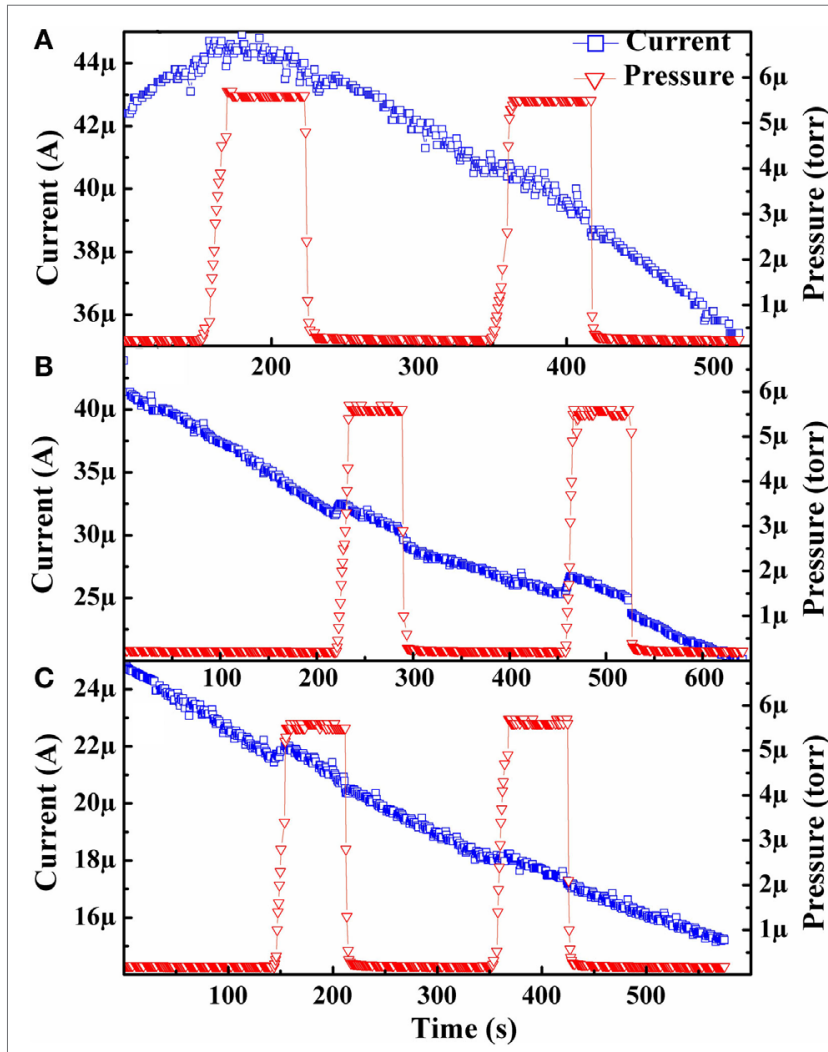

FIGURE 2 | Isothermal emission current behavior of a hydrogenated diamond in both vacuum and low-pressure hydrogen environment at: (A) $600^{\circ} \mathrm{C}$, (B) $625^{\circ} \mathrm{C}$, and (C) $650^{\circ} \mathrm{C}$. Only the first testing cycle for the hydrogenated sample is presented for publication purposes as a similar trend was seen for each test in the following two testing cycles. The current and pressure data are represented by blue squares and orange triangles, respectively.

hydrogenated sample, is that most of the surface carbon atoms are not bonded with a hydrogen atom and thus emission is not enhanced. The explanation for the overall increasing baseline current trend with time seen in Figure $\mathbf{1}$ is related to the deposition process conditions. That is, given that the diamond films are grown in a hydrogen-rich, methane-limited environment, a substantial amount of hydrogen is likely incorporated within the bulk of the diamond cathodes. As the emission current is monitored under increased heating, these hydrogen atoms diffuse from the interior of the diamond to the surface, bonding temporarily with surface carbon atoms. Should these bonds be made faster than the desorption of hydrogen, then the emission current would be enhanced as is seen in Figure 1. This explanation is further justified upon examination of the results shown in Figure 2, which come from a hydrogenated sample. This increasing trend is only temporary and after sufficient time, the emission current begins its predicted decreasing trend. The data collection for this study continued in this state rather than waiting for the current to begin its decreasing trend to ensure sufficiently high-emission current for analysis after the final test of cycle 3.

Isothermal testing of the sample post hydrogenation treatment demonstrated a similar enhancement of the emission 
current over vacuum baseline upon introduction of hydrogen to the as-grown sample but with much higher overall emission current values, as seen in Figure 2. These higher emission current levels following a hydrogenation treatment were anticipated based on previous work (Paxton et al., 2012a). As with Figure 1, only the results from the first testing cycle are presented; as results from the following two testing cycles demonstrated a similar behavior. In Figure 2, it can be observed that the baseline of the hydrogenated sample exhibits a decreasing trend that is attributed to desorption of the performance-enhancing hydrogen present from the hydrogen plasma treatment. This behavior is to be expected from the above reasoning for the increasing trend seen in the as-grown tests and from what has been seen in previous work examining the isothermal emission current from diamond films in vacuum (Paxton et al., 2012b, 2014).

In order to more clearly analyze the emission response directly attributable to the presence of externally introduced hydrogen for samples in both their as-grown and hydrogenated states, the transient nature of the background was subtracted from the current signal. This allowed for more accurate quantification of the current increase. A residual plot was taken of the data, which consisted of determining the trend of the background and subtracting each data point from the trend line, similar to a linear transformation. An example of this transformation is presented in Figure 3 for the $650^{\circ} \mathrm{C}$ test of the first as-grown testing cycle previously depicted in Figure 1C.

The magnitude of emission current increase due to the hydrogen environments determined from each residual plot was then compared against the baseline level immediately prior to the

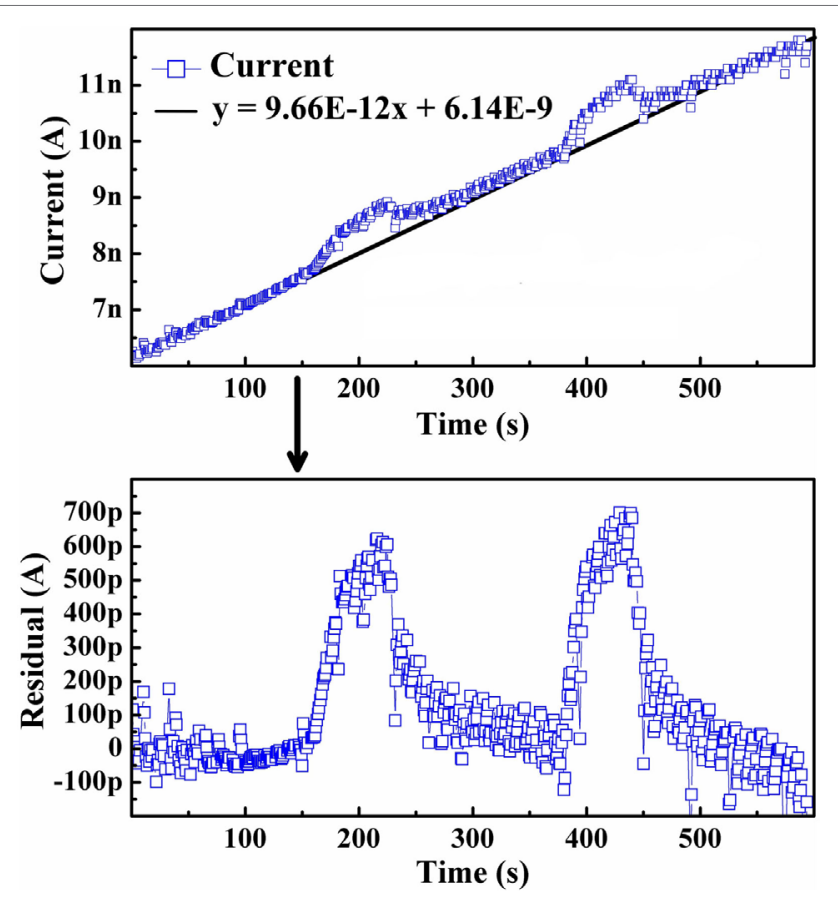

FIGURE 3 | Example of the residual plot performed for all isothermal tests. This technique was utilized to determine the magnitude of current increase due to the introduction of hydrogen. introduction of hydrogen. The resulting plot of this comparison, presented in Figure 4, indicates a definite relationship between the two. It can be seen that there exists a strong positive correlation between the current increase due to the introduction of hydrogen and the baseline current in vacuum. This trend was consistent across two samples (Sample A and Sample B in Figure 4) grown in separate batches confirming that this effect is reproducible.

Nitrogen-incorporated polycrystalline diamond cathodes were also tested in nitrogen gas environments in the same manner. The results from one of the testing runs on an as-grown diamond sample can be seen in Figure 5. Examination of the results confirmed the hypothesis that the thermionic emission performance of diamond cathodes in the presence of nitrogen gas would not be positively affected. The emission current clearly decreased from the baseline level established in high vacuum when the nitrogen was introduced into the chamber to a pressure of $\sim 5.5 \mu$ Torr $\left(\mathrm{N}_{2}\right.$ equivalent) measured at the pump.

The results collected in this study indicated that nitrogen gas was not a suitable candidate to improve the performance of diamond thermionic emitters. As both the molecular hydrogen and nitrogen experiments were performed at the same pressure and both gases have similar electron scattering cross-sections, the number of interactions of an electron with either species should also be similar. However, molecular nitrogen demonstrates a relatively high-magnitude negative electron affinity of $-1.8 \mathrm{eV}$ (Shilov, 2003) and high-dissociation energy of $9.75 \mathrm{eV}$ (Itikawa, 2006), preventing any excited/ionized species from interacting with the diamond. Similar results were seen in the previously discussed study by Koeck et al. that examined methane environments as a means to improve the performance of diamond cathodes. In that study, molecular nitrogen was also used as a control environment and it was found that such environments had no identifiable emission enhancement (Koeck et al., 2011).

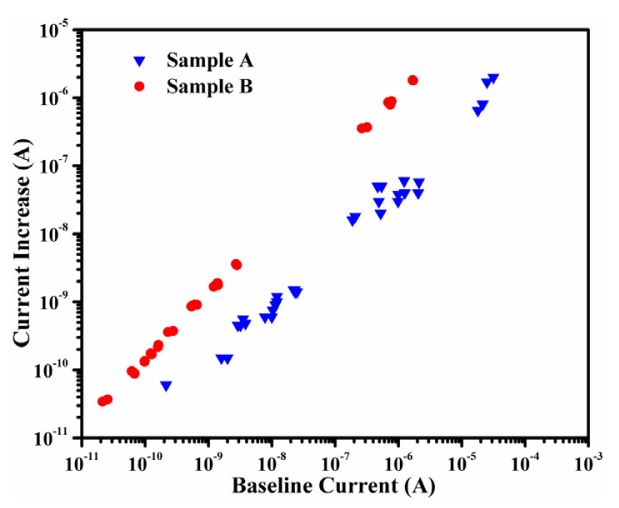

FIGURE 4 | Plot of the emission current increase due to the introduction of hydrogen as a function of the current in vacuum immediately prior to the introduction of hydrogen (baseline current) from Samples A and B. A clear linear relationship can be seen such that the magnitude of emission current improvement increases with increasing baseline current. The varying baseline current levels at each temperature are a result of this plot including data from the samples in both their as-grown and hydrogenated states. 


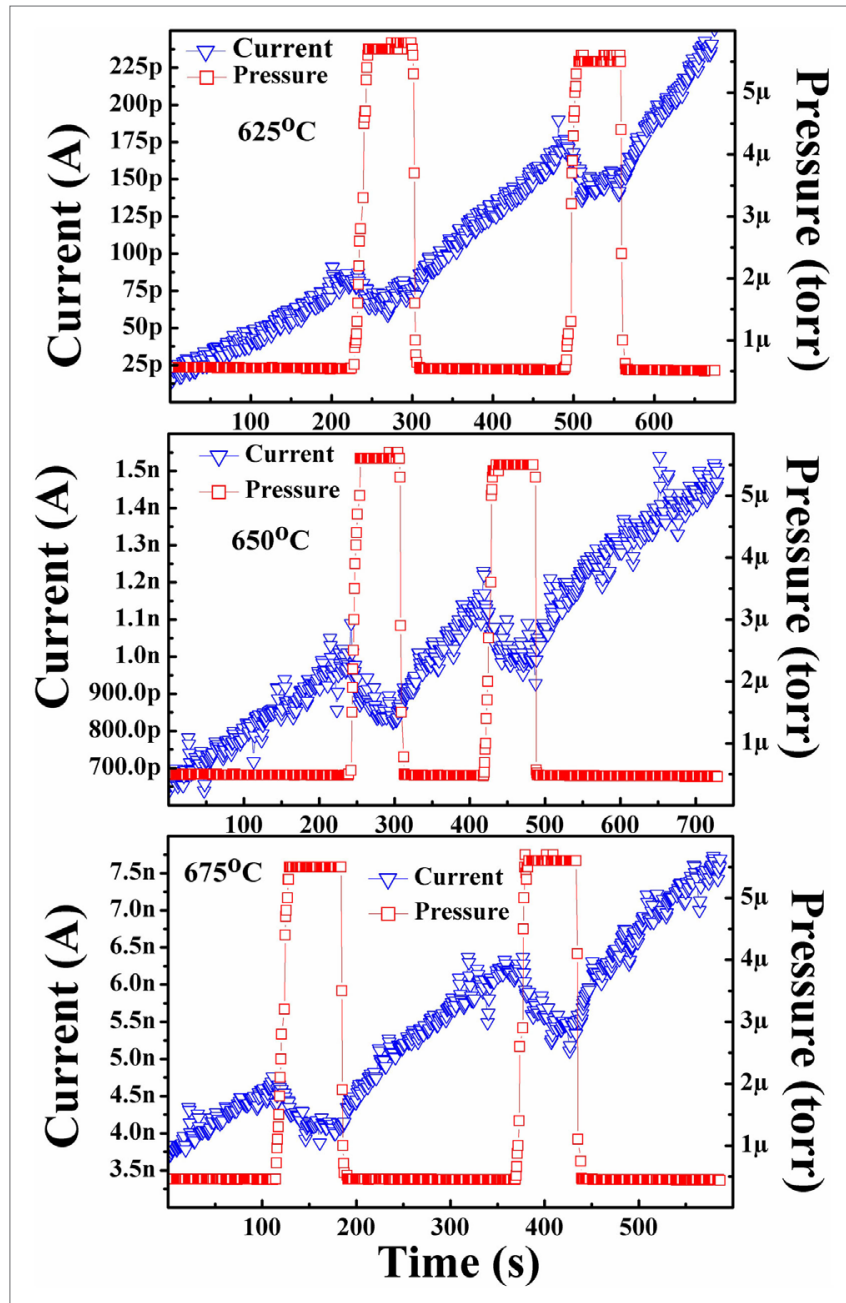

FIGURE 5 | Isothermal thermionic emission behavior of an as-grown nitrogen-incorporated diamond cathode in a nitrogen gaseous environment. It can be clearly seen that nitrogen had a negative effect on the emission performance from diamond cathodes which the authors attribute to the decreased mean-free path of electrons traveling from the cathode to the anode.

The observed decrease in emission current compared with vacuum found in the present study is attributed to the decrease in mean-free path caused by the scattering of the emitted electrons with the nitrogen molecules. While such electron collisions with molecular hydrogen should has have a similar likelihood as with molecular nitrogen, it is posited that such electron interactions with molecular hydrogen results in excited, ionized, or even dissociated hydrogen that leads to the observed emission current enhancement. There is also indication that Koeck et al. observed a similar decrease in emission current compared with vacuum as was seen in the present study but direct comparison is difficult due to the differing testing means. The results were consistent with the hypothesized effect $\mathrm{N}_{2}$ should have on the thermionic emission from diamond films. These nitrogen environment results also confirm that the positive response seen in the hydrogen environments is indeed solely due to the hydrogen and not an external variable.
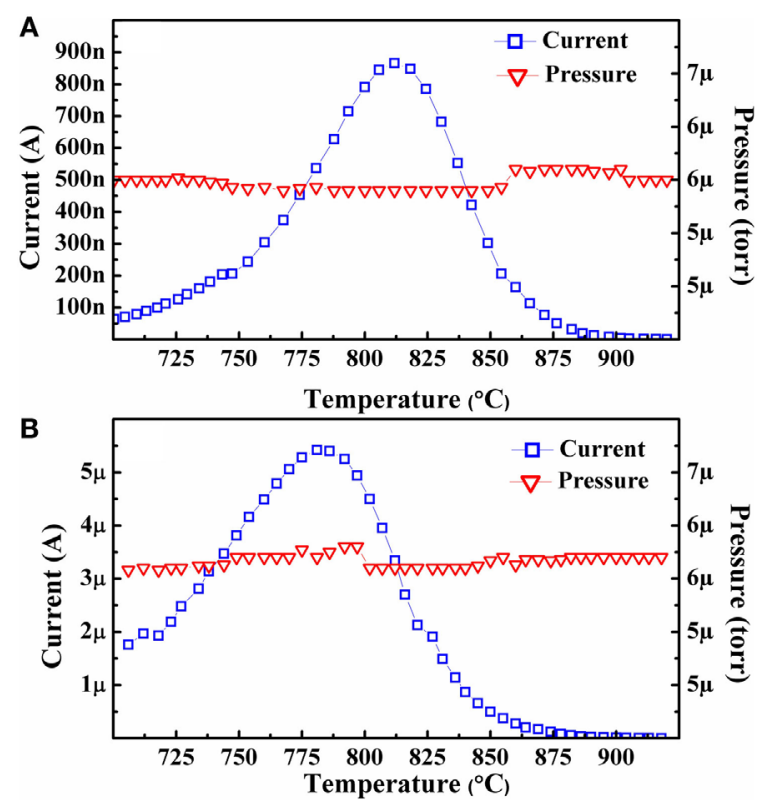

FIGURE 6 | Plots of the emission current versus temperature after completion of the isothermal experiments for Sample A in the (A) as-grown and $\mathbf{( B )}$ hydrogenated state.

\section{Isobaric Emission Testing Results}

As previously described, isobaric emission current versus temperature tests were also performed at a hydrogen environment pressure of $\sim 5.5 \times 10^{-6}$ Torr $\left(\mathrm{N}_{2}\right.$ equivalent) throughout the tests. Two such tests were performed after the third and final isothermal testing cycle for Sample A in both the as-grown and hydrogenated state. Both tests started at $\sim 700^{\circ} \mathrm{C}$ and continued to $\sim 900^{\circ} \mathrm{C}$.

From Figure 6A, it can be seen that the as-grown sample reached its maximum emission current levels at a temperature around $815^{\circ} \mathrm{C}$, which is slightly higher than the value seen in the previously reported as-grown emission tests (Paxton et al., 2012a). In addition to the increased temperature ceiling, operation in the hydrogen environment demonstrated an order of magnitude higher thermionic emission current at the same temperatures than a similar as-grown sample operating in vacuum (Paxton et al., 2012a). [Note: The emission area of all thermionic emission tests in the present and previously reported studies such as Paxton et al. (2012a) have been fixed at $4 \mathrm{~mm}^{2}$, allowing for direct comparison of performance between studies.] Figure 6B demonstrates that the maximum emission current was achieved at $\sim 780^{\circ} \mathrm{C}$. This is higher than the $\sim 700^{\circ} \mathrm{C}$ peak observed in the previous tests of similar hydrogenated diamond cathodes operating in vacuum (Paxton et al., 2012a).

\section{DISCUSSION}

The results indicate that the presence of hydrogen in the space between the cathode and anode has an enhancing effect on 
thermionic emission current. Attempts to fully understand this phenomenon are ongoing, but Figure 4 appears to rule out any thermal effects. According to Eq. 1, emission current should strictly be a function of temperature from a certain cathode. However, the hydrogenated diamond demonstrates significantly higher emission current than the as-grown diamond at the same temperature, as to be expected based on previously reported studies (Paxton et al., 2012a). It is these large baseline current variations (depending on the state of the diamond) that allows for thermal effects to be ruled out as the mechanism responsible for the observed response. If some interaction between the hydrogen and the diamond was occurring that was a function of temperature, then the same current increase would be observed at each temperature, regardless of cathode's baseline emission current levels. Observation of Figure 4 indicates that this is not the case. For instance, the data obtained at $625^{\circ} \mathrm{C}$ from Sample A in its as-grown state demonstrated an increase in current of $10^{-9} \mathrm{~A}$ over the $\sim 10^{-8} \mathrm{~A}$ baseline current. Conversely, when the baseline current at this temperature was $\sim 3 \times 10^{-5} \mathrm{~A}$ in the hydrogenated sample, the observed increase in current was over $10^{-6} \mathrm{~A}$. Thus, temperature does not appear to be the primary cause of the observed current improvements when operation occurs in a hydrogen gas environment.

The positive correlation between baseline current and emission current increase seen in Figure 4 suggests that the mechanism may be caused by the interaction of the thermionically emitted electrons with the hydrogen. Recall that the beneficial effects hydrogen has on the thermionic emission from diamond films are due to atomic hydrogen bonding with the surface carbon atoms, reducing the electron affinity. In the typical hydrogenation treatment, atomic hydrogen is formed from the dissociation of hydrogen due to electron collisions occurring in a plasma ambient. In the MPCVD reactor used for hydrogenation in the present study, microwaves stimulate electron oscillations which, at sufficient energies, collide with the molecular hydrogen, causing it to dissociate into atomic hydrogen (Regel and Wilcox, 2001). The atomic hydrogen then bonds with the diamond surface forming $\mathrm{C}-\mathrm{H}$ bonds that are favorable for thermionic emission. This same reaction may occur when the diamond thermionic cathodes are operating in a hydrogen environment. As electrons are emitted from the diamond and accelerated toward the anode, they can collide with the hydrogen present in the interelectrode gap. A portion of these emitted electrons will have sufficient energy to dissociate the hydrogen into atomic hydrogen ions. Some of this atomic hydrogen will migrate to the diamond cathode, attaching to dangling surface carbon bonds, thus enhancing emission current locally. If this reaction is occurring, then the magnitude of emission current enhancement should increase when more electrons are traversing the interelectrode gap (higher emission current), as was observed, Figure 4. When the hydrogen is shut off and evacuated from the chamber, these hydrogenated emission sites should then desorb following Arrhenius behavior and the emission current decrease to the baseline level. This trend can be seen in Figures 1 and 2. This represents one possible mechanism that could account for the observations made in the present study and appears to be consistent with previously reported work. Thus, additional studies should focus on determining how hydrogen promotes increased thermionic emission current from diamond films.

The results found in the present study also appear to be consistent with those found by the previously described study by Nemanič et al. (2014) that utilized a heated filament to produce excited, ionized, and/or dissociated hydrogen to enhance the emission current from diamond films. In that past study, Nemanič et al. observed a rapid increase in emission current over baseline when excited molecular hydrogen was introduced into the chamber followed by a rapid decay to baseline when the filament was turned off but with hydrogen still present in the chamber. This is particularly interesting as the present study was able to observe a similar effect but without the use of a heated filament. The observed current increases found in the present study represent a marked improvement over this past study in terms of practicality as a heated filament is not required to produce similar effects. Any observed influence of the molecular hydrogen environment without the assistance of the ion gauge observed by Nemanič et al. was attributed to a heated tungsten wire mounted behind the sample. The present study appears to contradict that explanation as the present study heated the samples directly with no other energy sources present in the chamber.

One such study by Wang et al. (2006) explored biasing as a means to modify the structural evolution of diamond films during hot-filament chemical vapor deposition (HFCVD). In the Wang et al. (2006) study, the discharge current between the heated filament and two different substrate holders was compared: (i) a molybdenum substrate holder and (ii) a diamond-coated, molybdenum substrate holder. Experiments performed in an HFCVD reactor consisted of negatively biasing each substrate holder with respect to the heated filament in a $\sim 35$ Torr hydrogen environment. The electric fields resulting from this bias were relatively low and on the same order as those used in the present study. Wang et al. observed a substantial increase in discharge current when using a diamond-coated, molybdenum substrate holder compared with an uncoated molybdenum holder. The authors attributed their observations to the negative electron affinity of the diamond surface, which promotes the emission of electrons (and thus discharge current) under the influence of the applied bias. Even when accounting for the small fields between the filament and the substrate holder, Wang et al. believed that the emitted electrons had sufficient energy to ionize the hydrogen following a collision. Due to the similar electric field strengths used in both studies, the same mechanism should be responsible for both the increased discharge current observed by Wang et al. and the improved thermionic emission current observed in the present study upon exposure to hydrogen environments.

It is yet to be determined whether or not hydrogen ions are indeed being produced through interaction of molecular hydrogen with the thermionically emitted electrons at the low-electric fields used in the present study. Limitations in in situ monitoring techniques make confirming this hypothesis difficult. However, if assumed true, there are two potential mechanisms that could enable the effects seen in the present study. The first deals with rehydrogenation of the diamond surface. The transient hydrogen ions could migrate to the diamond surface and fill the unoccupied carbon states, reducing the electron affinity, and increasing 
emission current. This is consistent with previously reported work (Paxton et al., 2012a). Another possibility is the reduction in work function due to the transient ions approaching the surface. A theoretical study by Go (2016) pertaining to ion-enhanced thermionic emission found that an approaching ion can significantly enhance emission current. This effect is magnified at low-electric fields (such as those used in the present study) due to the extended resonance time of the approaching ion. Both of these effects are possible in the presence of ionized hydrogen and would lead to the observed emission current enhancement in the present study. Work proceeds to identify the most likely candidate or whether the observed effect is due to a combination of both.

\section{CONCLUSION}

This work demonstrates that operation of diamond cathodes in hydrogen environments promotes improved thermionic emission current and increased temperature ceilings over typical vacuum operation. Analysis of the isothermal thermionic electron emission behavior at various temperatures from samples in both as-grown and hydrogenated states demonstrated a positive linear correlation between the magnitude of emission current increase over baseline (in a low-pressure hydrogen environment) and the baseline emission current (in vacuum). This trend is further verified as a second sample exhibited the same trend. Identical experiments in nitrogen environments did not demonstrate any beneficial effect, confirming that hydrogen is solely responsible the observed performance enhancement seen in the hydrogen environment experiments. Additionally, investigation of the isobaric performance of diamond cathodes further verified the

\section{REFERENCES}

Drabner, G., Poppe, A., and Budzikiewicz, H. (1990). The composition of the CH4 plasma. Int. J. Mass Spectrom. Ion Process. 97, 1-33. doi:10.1016/01681176(90)85037-3

Go, D. B. (2016). Analysis of thermionic and thermo-field emission in microscale gas discharges. J. Phys. D Appl. Phys. 49, 055206. doi:10.1088/0022$3727 / 46 / 3 / 035202$

Hatsopoulos, G. N. (1963). Transport effects in cesium thermionic converters. Proc. IEEE 51, 725-733. doi:10.1109/proc.1963.2265

Hernqvist, K. G. (1963). Analysis of the arc mode operation of the cesium vapor thermionic energy converter. Proc. IEEE 51, 748-754. doi:10.1109/ proc. 1963.2267

Itikawa, Y. (2006). Cross sections for electron collisions with nitrogen molecules. J. Phys. Chem. Ref. Data 35, 31-53. doi:10.1063/1.1937426

Jenkins, R. O. (1969). A review of thermionic cathodes. Vacuum 19, 353-359. doi:10.1016/s0042-207x(69)80077-1

Kataoka, M., Zhu, C., Koeck, F. A. M., and Nemanich, R. J. (2010). Thermionic electron emission from nitrogen-doped homoepitaxial diamond. Diamond Relat. Mater. 19, 110-113. doi:10.1016/j.diamond.2009.09.002

Koeck, F. A. M., Nemanich, R. J., Balasubramaniam, Y., Haenen, K., and Sharp, J. (2011). Enhanced thermionic energy conversion and thermionic emission from doped diamond films through methane exposure. Diamond Relat. Mater. 20, 1229-1233. doi:10.1016/j.diamond.2011.06.032

Maier, F., Ristein, J., and Ley, L. (2001). Electron affinity of plasma-hydrogenated and chemically oxidized diamond (100) surfaces. Phys. Rev. B 64, 165411. doi:10.1103/PhysRevB.64.165411

Nemanič, V., Žumer, M., Kovač, J., Koeck, F. A. M., and Nemanich, R. J. (2014). In situ reactivation of low-temperature thermionic electron emission from positive effect operation in a hydrogen environment has on thermionic emission. The temperature ceiling (i.e., the temperature at which emission current started to decrease) was distinctly higher when operating in a hydrogen gas environment compared with a vacuum environment. Furthermore, the as-grown sample demonstrated an order of magnitude increase in emission current as a result of hydrogen presence when compared with previously reported vacuum tests. To build on the results of this study, future studies will explore whether operation in higher hydrogen pressures results in further thermionic emission current enhancement; and, focus on quantifying the linear relationship between baseline current and hydrogen-related current increase.

\section{AUTHOR CONTRIBUTIONS}

WP devised the set of experiments, executed all tests, and assembled testing apparatus. SR assisted in analysis of results and assembly of manuscript. MB assisted in assembly and editing of manuscript. $\mathrm{MH}$ contributed in the design and assembly of the testing apparatus. JD advised on data collection and analysis.

\section{FUNDING}

Portions of this work were funded from NSF (EPS 1004083).

\section{SUPPLEMENTARY MATERIAL}

The Supplementary Material for this article can be found online at http://www.frontiersin.org/article/10.3389/fmech.2017.00018/ full\#supplementary-material. nitrogen doped diamond films by hydrogen exposure. Diamond Relat. Mater. 50, 151-156. doi:10.1016/j.diamond.2014.10.003

Paxton, W. F., Brooks, M. M., Howell, M., Tolk, N., Kang, W. P., and Davidson, J. L. (2014). Role of deuterium desorption kinetics on the thermionic emission properties of polycrystalline diamond films with respect to kinetic isotope effects. J. Appl. Phys. 115, 234904. doi:10.1063/ 1.4883185

Paxton, W. F., Howell, M., Kang, W. P., and Davidson, J. L. (2012a). Influence of hydrogen on the thermionic electron emission from nitrogen-incorporated polycrystalline diamond films. J. Vac. Sci. Technol. B Microelectron. Nanometer Struct. 30, 021202. doi:10.1116/1.3684982

Paxton, W. F., Steigerwald, A., Howell, M., Tolk, N., Kang, W. P., and Davidson, J. L. (2012b). The effect of hydrogen desorption kinetics on thermionic emission from polycrystalline chemical vapor deposited diamond. Appl. Phys. Lett. 101, 243509. doi:10.1063/1.4772069

Rasor, N. S. (1963). Emission physics of the thermionic energy converter. Proc. IEEE 51, 733-747. doi:10.1109/proc.1963.2266

Rasor, N. S. (1991). Thermionic energy conversion plasmas. IEEE Plasma Sci. Trans. 19, 1191-1208. doi:10.1109/27.125041

Regel, L. L., and Wilcox, W. R. (2001). Diamond film deposition by chemical vapor transport. Acta Astronaut. 48, 129-144. doi:10.1016/S00945765(00)00155-7

Richardson, O. W. (1903). The electrical conductivity imparted to a vacuum by hot conductors. Philos. Trans. R. Soc. Lond. A Contain. Papers Math. Phys. Character 201, 497-549. doi:10.1098/rsta.1903.0023

Shilov, A. E. (2003). Catalytic reduction of molecular nitrogen in solutions. Russian Chem. Bull. 52, 2555-2562. doi:10.1023/b:rucb.0000019873.81002.60

Suzuki, M., Ono, T., Sakuma, N., and Sakai, T. (2009). Low-temperature thermionic emission from nitrogen-doped nanocrystalline diamond films on n-type 
Si grown by MPCVD. Diamond Relat. Mater. 18, 1274-1277. doi:10.1016/j. diamond.2009.05.004

Tawara, H., Itikawa, Y., Nishimura, H., and Yoshino, M. (1990). Cross sections and related data for electron collisions with hydrogen molecules and molecular ions. J. Phys. Chem. Ref. Data 19, 617-636. doi:10.1063/1.555856

Wang, L., Yan, G. C., Li, Y., Cui, L., and Zhu, X. D. (2006). Bias-induced structural evolution of diamond films in plasma enhanced hot filament chemical vapour deposition with a two-step process. J. Phys. D Appl. Phys. 39, 3350. doi:10.1088/0022-3727/39/15/019

Wilson, V. C. (1959). Conversion of heat to electricity by thermionic emission. J. Appl. Phys. 30, 475-481. doi:10.1063/1.1702391

Witting, H. L., and Gyftopoulos, E. P. (1965). An ionization process in a low-energy cesium plasma. J. Appl. Phys. 36, 1328-1337. doi:10.1063/1.1714305
Conflict of Interest Statement: All financial, commercial, or other relationships that might be perceived by the academic community as representing a potential conflict of interest must be disclosed. If no such relationship exists, authors will be asked to confirm the following statement: The authors declare that the research was conducted in the absence of any commercial or financial relationships that could be construed as a potential conflict of interest.

Copyright (c) 2017 Paxton, Ravipati, Brooks, Howell and Davidson. This is an open-access article distributed under the terms of the Creative Commons Attribution License (CC BY). The use, distribution or reproduction in other forums is permitted, provided the original author(s) or licensor are credited and that the original publication in this journal is cited, in accordance with accepted academic practice. No use, distribution or reproduction is permitted which does not comply with these terms. 